TRANSACTIONS OF THE

AMERICAN MATHEMATICAL SOCIETY

Volume 354, Number 11, Pages 4345-4358

S 0002-9947(02)03066-0

Article electronically published on June 24, 2002

\title{
TILTING THEORY AND THE FINITISTIC DIMENSION CONJECTURES
}

\author{
LIDIA ANGELERI-HÜGEL AND JAN TRLIFAJ
}

Dedicated to Idun Reiten on the occasion of her sixtieth birthday

\begin{abstract}
Let $R$ be a right noetherian ring and let $\mathcal{P}<\infty$ be the class of all finitely presented modules of finite projective dimension. We prove that findim $R=n<\infty$ iff there is an (infinitely generated) tilting module $T$ such that $\operatorname{pd} T=n$ and $T^{\perp}=\left(\mathcal{P}^{<\infty}\right)^{\perp}$. If $R$ is an artin algebra, then $T$ can be taken to be finitely generated iff $\mathcal{P}<\infty$ is contravariantly finite. We also obtain a sufficient condition for validity of the First Finitistic Dimension Conjecture that extends the well-known result of Huisgen-Zimmermann and Smalø.
\end{abstract}

For a ring $R$, the little finitistic dimension, findim $R$, is defined as the supremum of the projective dimensions attained on the category of all finitely generated right $R$-modules of finite projective dimension. The big finitistic dimension, Findim $R$, is defined correspondingly on the category of arbitrary right $R$-modules of finite projective dimension.

It is well known that these dimensions may be infinite. Moreover, they do not coincide in general. For example, if $R$ is a commutative local noetherian ring, we know by classical results of Bass, Gruson and Raynaud, and Auslander and Buchweitz that Findim $R$ is just the Krull dimension and findim $R$ coincides with the depth of $R$. So in this case, $\operatorname{Fin} \operatorname{dim} R=\operatorname{findim} R$ if and only if $R$ is a CohenMacaulay ring.

In the case when $R$ is a finite-dimensional algebra over a field, the little and the big finitistic dimensions may also differ; that is, the First Finitistic Dimension Conjecture fails. The first example of this phenomenon was given by HuisgenZimmermann in 1992. Later, Smalø showed that the difference between the two dimensions can be arbitrarily big [18. However, it is still an open question, known as the Second Finitistic Dimension Conjecture, whether the little finitistic dimension of a finite-dimensional algebra $R$ is always finite.

Positive answers are known in some particular cases. For instance, it was shown by Auslander and Reiten [5] that findim $R$ is finite provided that the category $\mathcal{P}<\infty$ of all finitely presented modules of finite projective dimension is contravariantly finite in $\bmod R$. It is then enough to consider the projective dimensions of the right $\mathcal{P}^{<\infty}$-approximations of the (finitely many) simple modules; the projective dimension of any other finitely generated module is either infinite or bounded by the maximum of these numbers. Huisgen-Zimmermann and Smalø [13] later extended

Received by the editors July 13, 2001 and, in revised form, February 7, 2002.

2000 Mathematics Subject Classification. Primary 16E10, 16E30, 16G10.

Research supported by an HWP-grant of LMU Munich and by grants GAČR 201/00/0766 and MSM 113200007. 
this result to arbitrary modules by showing that every module of finite projective dimension is then a direct limit of modules in $\mathcal{P}<\infty$. In this way, they proved that contravariant finiteness of $\mathcal{P}<\infty$ implies Findim $R=$ findim $R$.

The idea of Auslander and Reiten can be extended beyond the particular case when $\mathcal{P}<\infty$ is contravariantly finite. The point is that the larger class $\mathcal{A}=$ ${ }^{\perp}\left(\left(\mathcal{P}^{<\infty}\right)^{\perp}\right)$ always provides for right approximations. If $R$ is right artinian, then the little finitistic dimension can be computed as the maximum of the projective dimensions of the right $\mathcal{A}$-approximations of the (finitely many) simple $R$-modules, [20.

In the present paper, we extend and refine the results mentioned above by providing sufficient conditions for validity of the First and the Second Finitistic Dimension Conjectures. The novel idea here is to study the complete cotorsion pair cogenerated by $\mathcal{P}<\infty$ by means of the theory of (infinitely generated) tilting modules.

We show for any right noetherian $\operatorname{ring} R$ that findim $R<\infty$ if and only if the Ext-orthogonal class $\left(\mathcal{P}^{<\infty}\right)^{\perp}$ equals $T^{\perp}$ where $T$ is an (infinitely generated) tilting module in the sense of 1 . Moreover, findim $R=\operatorname{pd} T$, and the artin algebras $R$ for which $T$ can be chosen finitely presented are precisely those with $\mathcal{P}^{<\infty}$ contravariantly finite.

We also prove that modules of finite projective dimension coincide with direct summands of $\mathcal{P}^{<\infty}$-filtered modules if and only if the category $\operatorname{Add} T$ is closed under cokernels of monomorphisms. We thus obtain a sufficient condition for Findim $R=$ findim $R$ that extends the result of Huisgen-Zimmermann and Smalø mentioned above.

The paper is organized as follows. In Section 1, we review basic properties of Ext-orthogonal classes, complete cotorsion pairs and tilting modules following [1] and [9. Our main results for the right coherent case are proved in Sections 2 and 3. Section 4 treats, in more detail, the particular case of artin algebras.

This research was done during a visit by the first author to MFF UK in Prague in March 2001 and supported by an exchange program with LMU Munich. The first author would like to thank the Department of Algebra of MFF UK for its hospitality.

We wish to thank Birge Huisgen-Zimmermann and the referee for interesting comments on an earlier version of this paper.]

\section{Cotorsion pairs, tilting MOdules, AND APPROXimations}

For an arbitrary ring $R$ we denote by $\operatorname{Mod} R$ the category of all (right) $R$-modules, and by $\bmod R$ the subcategory of $\operatorname{Mod} R$ consisting of all finitely presented modules.

Let $M \in \operatorname{Mod} R$ and let

$$
\Theta: \cdots \stackrel{f_{i+2}}{\longrightarrow} P_{i+1} \stackrel{f_{i+1}}{\longrightarrow} P_{i} \stackrel{f_{i}}{\longrightarrow} \ldots \stackrel{f_{1}}{\longrightarrow} P_{0} \stackrel{f_{0}}{\longrightarrow} M \longrightarrow 0
$$

be a projective resolution of $M$. Let $i<\omega$. Then the module $\operatorname{Im}\left(f_{i}\right)$ is called the $i$-th syzygy of $M$ in $\Theta$. Dually, the $i$-th cosyzygy of $M$ is defined in an injective coresolution of $M$. Denote by $\boldsymbol{\Omega}^{i}(\boldsymbol{M})$ the class of the $i$-th syzygies occurring in

\footnotetext{
${ }^{1}$ Added in proof: In this paper, we have not considered the obvious dual questions concerning relations between cotilting modules and the finitistic injective dimensions. In fact, if the cotilting modules are assumed to be pure-injective, then the approximation theory works fine and some of our results can be dualized. For more on this topic, we refer to the recent manuscript of Buan, Krause and Solberg, "On the lattice of cotilting modules".
} 
all projective resolutions of $M$. Similarly, $\boldsymbol{\Omega}^{-i}(\boldsymbol{M})$ denotes the class of the $i$-th cosyzygies occurring in all injective coresolutions of $M$.

The ring $R$ is called right coherent provided that all finitely generated right ideals of $R$ are finitely presented (equivalently, all finitely generated submodules of finitely presented right modules are finitely presented). If $R$ is right coherent and $M$ is finitely presented, then we will consider only projective resolutions consisting of finitely presented modules; so all the syzygies of $M$ will be finitely presented in this case.

Let $\mathcal{M} \subseteq \operatorname{Mod} R$. For an integer $i$, define $\boldsymbol{\Omega}^{i}(\mathcal{M})=\bigcup_{M \in \mathcal{M}} \Omega^{i}(M)$. We say that $\mathcal{M}$ is syzygy-closed, provided that $\Omega^{1}(\mathcal{M}) \subseteq \mathcal{M}$. Similarly, $\mathcal{M}$ is cosyzygyclosed provided that $\Omega^{-1}(\mathcal{M}) \subseteq \mathcal{M}$. Of course, if $\mathcal{M}$ is syzygy-closed, then all the classes $\Omega^{i}(\mathcal{M}), i<\omega$, are syzygy-closed.

For each $0<i<\omega$, we set

$$
\mathcal{M}^{\perp_{i}}=\left\{X \in \operatorname{Mod} R \mid \operatorname{Ext}_{R}^{i}(M, X)=0 \text { for all } M \in \mathcal{M}\right\}
$$

and

$$
\mathcal{M}^{\perp}=\bigcap_{0<i<\omega} \mathcal{M}^{\perp_{i}}
$$

Dually, we define ${ }^{\perp_{i}} \mathcal{M}=\left\{X \in \operatorname{Mod} R \mid \operatorname{Ext}_{R}^{i}(X, M)=0\right.$ for all $\left.M \in \mathcal{M}\right\}$, and ${ }^{\perp} \mathcal{M}=\bigcap_{0<i<\omega}{ }^{\perp_{i}} \mathcal{M}$.

Finally, recall that $\mathcal{M}$ is resolving (coresolving) if it is closed under extensions, kernels of epimorphisms (cokernels of monomorphisms), and it contains all projective (injective) modules. For example, for any $\mathcal{M} \subseteq \operatorname{Mod} R$, the class $\mathcal{M}^{\perp}$ is coresolving, and ${ }^{\perp} \mathcal{M}$ is resolving.

Obviously, any resolving class is syzygy-closed, and any coresolving class is cosyzygy-closed. If $\mathcal{M}$ is a syzygy-closed class of modules, then $\mathcal{M}^{\perp_{1}}=\mathcal{M}^{\perp}$ is coresolving, and ${ }^{\perp}\left(\mathcal{M}^{\perp}\right)={ }^{\perp_{1}}\left(\mathcal{M}^{\perp}\right)$ is resolving. Similarly, if $\mathcal{M}$ is cosyzygy-closed, then ${ }^{{ }^{1}} \mathcal{M}={ }^{\perp} \mathcal{M}$ is resolving, and $\left({ }^{\perp} \mathcal{M}\right)^{\perp}=\left({ }^{\perp} \mathcal{M}\right)^{\perp_{1}}$ is coresolving.

Next, we recall the notion of a cotorsion pair. This is the analog of the classical (nonhereditary) torsion pair where Hom is replaced by $\mathrm{Ext}^{1}$ :

Definition. Let $\mathcal{A}, \mathcal{B} \subseteq \operatorname{Mod} R$ be classes of modules. Then $(\mathcal{A}, \mathcal{B})$ is a cotorsion pair if $\mathcal{A}={ }^{\perp_{1}} \mathcal{B}$ and $\mathcal{B}=\mathcal{A}^{\perp_{1}}$.

For any class $\mathcal{C} \subseteq \operatorname{Mod} R$, we have the cotorsion pair $\left({ }^{\perp_{1}}\left(\mathcal{C}^{\perp_{1}}\right), \mathcal{C}^{\perp_{1}}\right)$, called the cotorsion pair cogenerated by $\mathcal{C}$.

Now, if $\mathcal{C}$ is syzygy-closed, then the cotorsion pair cogenerated by $\mathcal{C}$ coincides with $\left({ }^{\perp}\left(\mathcal{C}^{\perp}\right), \mathcal{C}^{\perp}\right)$. In particular, if $C$ is a module and $\mathcal{X}=C^{\perp}$, then $\left({ }^{\perp} \mathcal{X}, \mathcal{X}\right)$ is the cotorsion pair cogenerated by the set $\mathcal{S}=\left\{S_{i} \mid i<\omega\right\}$ where $S_{i}$ denotes the $i$-th syzygy in a fixed projective resolution of $C$.

We will also need several concepts concerning approximations of modules.

Let $\mathcal{M} \subseteq \operatorname{Mod} R$ and $A \in \operatorname{Mod} R$. A morphism $f \in \operatorname{Hom}_{R}(A, X)$ with $X \in$ $\mathcal{M}$ is an $\mathcal{M}$-preenvelope of $A$ provided that the abelian group homomorphism $\operatorname{Hom}_{R}(f, M): \operatorname{Hom}_{R}(X, M) \longrightarrow \operatorname{Hom}_{R}(A, M)$ is surjective for each $M \in \mathcal{M}$. An $\mathcal{M}$-preenvelope $f \in \operatorname{Hom}_{R}(A, X)$ of $A$ is special if $f$ is a monomorphism and Coker $f \in{ }^{\perp_{1}} \mathcal{M}$. Note that by the observation above, the cokernel of any special $\mathcal{M}$-preenvelope is actually contained in ${ }^{\perp} \mathcal{M}$ provided that $\mathcal{M}$ is coresolving.

The notion of a (special) $\mathcal{M}$-precover is defined dually?

\footnotetext{
${ }^{2}$ In the representation theory of artin algebras, the usual terminology for a preenvelope and a precover $($ in $\bmod R)$ is a left, respectively a right, approximation.
} 
The basic relation between cotorsion pairs and approximations goes back to Salce [17]. It may be viewed as a substitute for the nonexistence of a duality for arbitrary modules:

Lemma 1.1 ([17, Corollary 2.4.]). Let $R$ be a ring and $(\mathcal{A}, \mathcal{B})$ a cotorsion pair. The following are equivalent:

(1) every module has a special $\mathcal{A}$-precover;

(2) every module has a special $\mathcal{B}$-preenvelope.

In this case, the cotorsion pair $(\mathcal{A}, \mathcal{B})$ is called complete.

Remark 1.2 ([5, p. 125]). If we restrict all these notions to the category of all finitely presented modules, Lemma 1.1 remains true provided that $R$ is right coherent and injective envelopes of finitely presented modules are finitely presented (this occurs in case $R$ is an artin algebra, for example).

The key fact about cotorsion pairs — first proved in 9], and motivated by [10 says that any cotorsion pair $(\mathcal{A}, \mathcal{B})$ cogenerated by a set of modules $\mathcal{S}$ is complete. Moreover, there is a description of the class $\mathcal{A}$ in terms of the modules from $\mathcal{S}$. In order to formulate it, we need one more notion:

Let $\sigma$ be an ordinal. A chain of modules, $\left(M_{\alpha} \mid \alpha \leq \sigma\right)$, is continuous provided that $M_{\alpha} \subseteq M_{\alpha+1}$ for all $\alpha<\sigma$ and $M_{\alpha}=\bigcup_{\beta<\alpha} M_{\beta}$ for all limit ordinals $\alpha \leq \sigma$.

Let $M$ be a module and $\mathcal{C}$ a class of modules. Then $M$ is $\mathcal{C}$-filtered provided that there is a continuous chain $\left(M_{\alpha} \mid \alpha \leq \sigma\right)$ consisting of submodules of $M$ such that $M=M_{\sigma}$, and each of the modules $M_{0}, M_{\alpha+1} / M_{\alpha}(\alpha<\sigma)$, is isomorphic to an element of $\mathcal{C}$.

Theorem 1.3. Let $R$ be a ring and $\mathcal{S} \subseteq \operatorname{Mod} R$ a set of modules. Let $(\mathcal{A}, \mathcal{B})$ be the cotorsion pair cogenerated by $\mathcal{S}$.

(1) [9, Theorem 10] $(\mathcal{A}, \mathcal{B})$ is complete.

(2) [19, Theorem 2.2] If $R \in \mathcal{S}$, then $\mathcal{A}$ consists of all direct summands of $\mathcal{S}$ filtered modules. Moreover, for each $A \in \mathcal{A}$ there exist $C \in \mathcal{A} \cap \mathcal{B}$ and an $\mathcal{S}$-filtered module $D$ such that $D=A \oplus C$.

We will also use the following well-known homological facts:

Lemma 1.4 ([8, Theorem 7.3.4]). Let $R$ be a ring. Let $M$ and $N$ be modules and $0<n<\omega$. Assume that $M$ is ${ }^{\perp_{n}} N$-filtered. Then $M \in{ }^{\perp_{n}} N$.

Lemma 1.5 ([8, Lemma 10.2.4]). Let $R$ be a right coherent ring, $0<n<\omega$, let $M$ be a finitely presented module, and let $\left(N_{\alpha}, f_{\alpha \beta} \mid \alpha<\beta \in I\right)$ be a direct system of modules. Then

$$
\operatorname{Ext}_{R}^{n}\left(M, \lim _{\longrightarrow} N_{\alpha}\right) \cong \underset{\lim }{\longrightarrow} \operatorname{Ext}_{R}^{n}\left(M, N_{\alpha}\right) .
$$

In particular, $\mathcal{M}^{\perp_{n}}$ and $\mathcal{M}^{\perp}$ are closed under arbitrary direct limits for any class of finitely presented modules $\mathcal{M}$.

Lemma 1.6 ([4, Chap 1, Proposition 10.1]). Let $R$ be a ring, $0<n<\omega$, let $M$ be a pure-injective module, and let $\left(N_{\alpha}, f_{\alpha \beta} \mid \alpha<\beta \in I\right)$ be a direct system of modules. Then

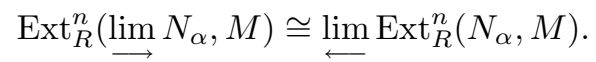

In particular, ${ }^{\perp_{n}} \mathcal{M}$ and $\perp^{\perp} \mathcal{M}$ are closed under arbitrary direct limits for any class of pure-injective modules $\mathcal{M}$. 
Finally, we recall several notions and results from tilting theory.

Let $R$ be a ring. For a class of modules $\mathcal{M} \subseteq \operatorname{Mod} R$, denote by Add $\mathcal{M}$ (respectively, add $\mathcal{M}$ ) the class consisting of all modules isomorphic to direct summands of (finite) direct sums of elements of $\mathcal{M}$.

Definition. Let $R$ be a ring. A module $M$ is a tilting module provided that

(T1) $\operatorname{pd} M<\infty$;

(T2) $\operatorname{Ext}_{R}^{i}\left(M, M^{(I)}\right)=0$ for each $i>0$ and all sets $I$;

(T3) there is a long exact sequence $0 \longrightarrow R_{R} \longrightarrow M_{0} \longrightarrow \cdots \longrightarrow M_{r} \longrightarrow 0$ where $r \geq 0$ and $M_{i} \in \operatorname{Add} M$ for each $0 \leq i \leq r$.

Observe that if $M$ is a tilting module and $\mathcal{X}=M^{\perp}$, then $\left({ }^{\perp} \mathcal{X}, \mathcal{X}\right)$ is a complete cotorsion pair. Moreover, the long exact sequence of (T3) can be obtained from short exact sequences given by special $\mathcal{X}$-preenvelopes. By Theorem 1.3(2) or [1, 2.2], the cokernels of these preenvelopes have projective dimension bounded by $n=\operatorname{pd} M$, and by dimension shifting, the $(n+1)$-th sequence splits. So we can assume that $r \leq \operatorname{pd} M$ in condition (T3).

Tilting modules generalize the finite-dimensional tilting modules studied in the representation theory of finite-dimensional algebras (cf. [11, 16] et al.) as well as the infinitely generated tilting modules of projective dimension $\leq 1$ studied in [6]

We point out some important properties.

Lemma 1.7. Let $R$ be a ring, $M \in \operatorname{Mod} R$ a tilting module and $\mathcal{X}=M^{\perp}$.

(a) For each $X \in \mathcal{X}$, there exists a short exact sequence $0 \longrightarrow K \longrightarrow M^{\prime} \longrightarrow$ $X \longrightarrow 0$ with $M^{\prime} \in \operatorname{Add} M$ and $K \in \mathcal{X}$.

(b) Add $M=\mathcal{X} \cap{ }^{\perp} \mathcal{X}$.

(c) For each $X \in \mathcal{X}$ of projective dimension $n$ there is a long exact sequence

$$
0 \longrightarrow M_{n+1} \longrightarrow M_{n} \longrightarrow M_{n-1} \longrightarrow \cdots \longrightarrow M_{0} \longrightarrow X \longrightarrow 0
$$

with $M_{i} \in$ Add $M$ for all $i=0, \ldots, n+1$.

Proof. (a) and (b) are shown in 1, 2.3 and 2.4]. Part (c) is only implicit in [1, 4.1], and so we present a full proof here.

Let $X \in \mathcal{X}$ be a module of projective dimension $n$. Iterating (a), we obtain a long exact sequence $0 \longrightarrow K_{n} \longrightarrow M_{n} \stackrel{f_{n}}{\longrightarrow} M_{n-1} \longrightarrow \cdots \stackrel{f_{1}}{\longrightarrow} M_{0} \stackrel{f_{0}}{\longrightarrow} X \longrightarrow 0$ with $M_{i} \in \operatorname{Add} M$ and $K_{i}=\operatorname{Ker} f_{i} \in M^{\perp}$ for $i=0, \ldots, n$. Since $\operatorname{pd} X \leq n$, by dimension shifting we have $\operatorname{Ext}_{R}^{1}\left(K_{n-1}, K_{n}\right) \cong \operatorname{Ext}_{R}^{n+1}\left(X, K_{n}\right)=0$ and so $K_{n} \in$ $\operatorname{Add} M$.

The relationship between tilting modules and existence of preenvelopes is established by the following fundamental result. Again, it is a common generalization of the classical finite-dimensional case [5] and the case of infinitely generated modules of projective dimension at most one [3].

Theorem 1.8 ([1, 4.1]). Let $R$ be a ring and $n<\omega$. Let $\mathcal{X} \subseteq \operatorname{Mod} R$ be a class of modules closed under direct summands, cokernels of monomorphisms, and such that $\mathcal{X} \cap{ }^{\perp} \mathcal{X}$ is closed under coproducts. The following are equivalent:

(1) There exists a tilting module $M$ of projective dimension at most $n$ such that $\mathcal{X}=M^{\perp}$.

(2) Every module has a special $\mathcal{X}$-preenvelope, and all modules in $\perp \mathcal{X}$ have projective dimension at most $n$. 
The proof of Theorem 1.8 is constructive: assuming (2), one obtains the tilting module $M$ in the form $M=\bigoplus_{i \leq n} M_{i}$ where

$$
0 \longrightarrow R \stackrel{f_{0}}{\longrightarrow} M_{0} \stackrel{f_{1}}{\longrightarrow} M_{1} \longrightarrow \cdots \stackrel{f_{n}}{\longrightarrow} M_{n} \longrightarrow 0
$$

is a long exact sequence obtained by an iteration of special $\mathcal{X}$-preenvelopes of the module $R$, of Coker $f_{0}$, etc.

Finally, observe that if $M$ is a tilting module, then every module $T$ with AddT $=\operatorname{Add} M$ is again a tilting module with $T^{\perp}=M^{\perp}$.

\section{A tilting MOdUle MEASURING THE LitTle Finitistic Dimension}

For a ring $R$, we denote by $\mathcal{P}$ the class of all modules of finite projective dimension and let $\mathcal{P}<\infty=\mathcal{P} \cap \bmod -R$ be the class of all finitely presented modules of finite projective dimension.

Moreover, for $n<\omega$, we let $\mathcal{P}_{\boldsymbol{n}}$ be the class of all modules of projective dimension at most $n$, and set $\mathcal{P}_{n}^{<\infty}=\mathcal{P}_{n} \cap \bmod R$. Clearly, $\mathcal{P}=\bigcup_{n<\omega} \mathcal{P}_{n}$ and $\mathcal{P}^{<\infty}=$ $\bigcup_{n<\omega} \mathcal{P}_{n}^{<\infty}$. Note that the classes $\mathcal{P}$ and $\mathcal{P}_{n}(n<\omega)$ are syzygy-closed, and the same is true of the classes $\mathcal{P}<\infty$ and $\mathcal{P}_{n}^{<\infty}(n<\omega)$ provided that $R$ is right coherent.

Denote by $(\mathcal{A}, \mathcal{B})$ the cotorsion pair cogenerated by the class $\mathcal{P}<\infty$. For each $n<\omega$, let $\left(\mathcal{A}_{n}, \mathcal{B}_{n}\right)$ be the cotorsion pair cogenerated by the class $\mathcal{P}_{n}^{<\infty}$.

By Theorem 1.3 and Lemma 1.4, all these cotorsion pairs are complete, and $\mathcal{A}_{n} \subseteq \mathcal{P}_{n}$.

If $R$ is right coherent, it follows from Lemma 1.5 that $\mathcal{B}$ and all the classes $\mathcal{B}_{n}$ are definable in the sense of [7] $\left.\S 2.3\right]$. In particular, they are determined by their (indecomposable) pure-injective modules. Furthermore, the modules in $\mathcal{A}_{n}$ are direct limits of finitely presented modules in $\mathcal{P}_{n}$, and the modules in $\mathcal{A}$ are direct limits of finitely presented modules in $\mathcal{P}$, as shown below.

Lemma 2.1. Let $R$ be right coherent. Then every module in $\mathcal{A}$ is a direct limit of modules in $\mathcal{P}<\infty$.

Proof. Denote by $\mathcal{D}$ the class of all direct limits of modules in $\mathcal{P}<\infty$. Since $\mathcal{P}<\infty$ consists of finitely presented modules, the modules $D$ in $\mathcal{D}$ are characterized by the property that every homomorphism $h: F \rightarrow D$ where $F$ is finitely presented factors through a module in $\mathcal{P}<\infty$; see [15, 2.1]. This implies first of all that $\mathcal{D}$ is closed under direct limits, and thus under direct summands as well.

Moreover, it implies that $\mathcal{D}$ is closed under extensions. In fact, let $0 \longrightarrow A \stackrel{f}{\longrightarrow}$ $B \stackrel{g}{\longrightarrow} C \longrightarrow 0$ be an exact sequence with $A$ and $C$ in $\mathcal{D}$, and $h: F \rightarrow B$ be a homomorphism where $F$ is finitely presented. Then $g h$ factors through a module $P^{\prime \prime}$ in $\mathcal{P}^{<\infty}$; so there are maps $\sigma^{\prime \prime}: F \rightarrow P^{\prime \prime}$ and $\tau^{\prime \prime}: P^{\prime \prime} \rightarrow C$ such that $g h=\tau^{\prime \prime} \sigma^{\prime \prime}$. Let $p: R^{n} \rightarrow P^{\prime \prime}$ be an epimorphism with an integer $n \geq 0$, and let $p^{\prime}: R^{n} \rightarrow B$ satisfy $g p^{\prime}=\tau^{\prime \prime} p$. Denote by $g^{\prime \prime}: F \oplus R^{n} \rightarrow P^{\prime \prime}$ the coproduct map induced by $\sigma^{\prime \prime}$ and $p$, and by $h^{\prime \prime}: F \oplus R^{n} \rightarrow B$ the coproduct map induced by $h$ and $p^{\prime}$. We then obtain a commutative diagram

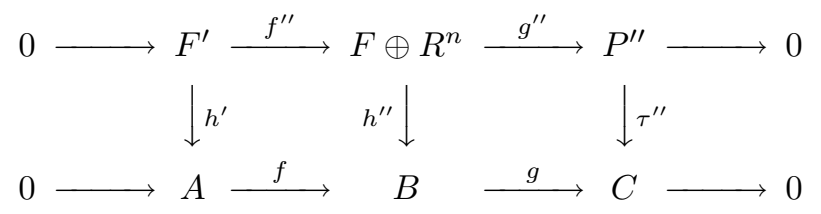


Since $P^{\prime \prime}$ and $F \oplus R^{n}$ are finitely presented and the $\operatorname{ring} R$ is right coherent, we deduce that $F^{\prime}$ is finitely presented. Thus, $h^{\prime}$ factors through a module $P^{\prime}$ in $\mathcal{P}<\infty$; so there are maps $\sigma^{\prime}: F^{\prime} \rightarrow P^{\prime}$ and $\tau^{\prime}: P^{\prime} \rightarrow A$ such that $h^{\prime}=\tau^{\prime} \sigma^{\prime}$. In the push-out diagram

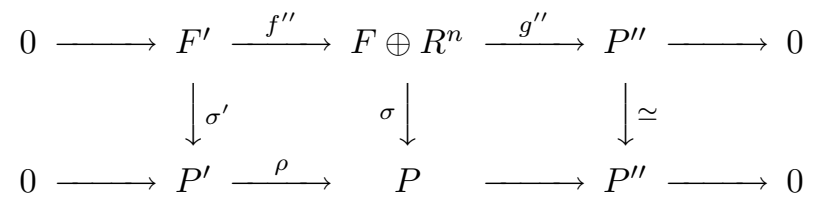

we have that $P$ belongs to $\mathcal{P}^{<\infty}$, and by the push-out property, we obtain a map $\tau: P \rightarrow B$ such that $\tau \sigma=h^{\prime \prime}$, hence $\left.\tau \sigma\right|_{F}=h$. This shows that $h$ factors through a module $P$ in $\mathcal{P}^{<\infty}$. Therefore, $B$ belongs to $\mathcal{D}$.

So, we can conclude by transfinite induction that every $\mathcal{P}<\infty$-filtered module belongs to $\mathcal{D}$. Thus, if $A$ is in $\mathcal{A}$, it follows from Theorem[1.3(2) that $A$ is a direct summand of a module $D$ in $\mathcal{D}$, and therefore $A$ belongs to $\mathcal{D}$.

The close relation of these classes to tilting theory comes from the following observation:

Lemma 2.2. Let $n<\omega$. Let $R$ be a right coherent ring and $\mathcal{S}$ a syzygy-closed class of finitely presented modules. Denote by $(\mathcal{U}, \mathcal{V})$ the cotorsion pair cogenerated by $\mathcal{S}$. The following are equivalent:

(1) $\mathcal{U} \subseteq \mathcal{P}_{n}$;

(2) there exists a tilting module $T$ of projective dimension at most $n$ such that $\mathcal{V}=T^{\perp}$.

Proof. Since $\mathcal{S}$ is syzygy-closed, $\mathcal{V}$ is coresolving and $\mathcal{U}={ }^{\perp} \mathcal{V}$. Moreover, $\mathcal{V}$ is closed under arbitrary direct limits by Lemma 1.5 , so $\mathcal{U} \cap \mathcal{V}$ is closed under coproducts. Finally, Theorem 1.3(1) implies that $\mathcal{V}$ provides for special preenvelopes. The claim now follows from Theorem 1.8 .

We now apply our observation to the cotorsion pairs considered above.

Proposition 2.3. Let $R$ be a right coherent ring. For any $n<\omega$, there is a tilting module $T_{n}$ of projective dimension at most $n$ such that $\mathcal{B}_{n}=T_{n}^{\perp}$. If $R$ is right noetherian and findim $R \geq n$, then $T_{n}$ has projective dimension $n$.

Proof. The first part is just Lemma 2.2 for $\mathcal{S}=\mathcal{P}_{n}^{<\infty}$.

Assume that $R$ is right noetherian with $\operatorname{fin} \operatorname{dim} R \geq n$. So, there is a finitely presented module $M$ of projective dimension $n$. Assume that there is a tilting module $T \in \mathcal{P}_{n-1}$ with $\mathcal{B}_{n}=T^{\perp}$. By Lemma 2.2, we then have $\mathcal{A}_{n} \subseteq \mathcal{P}_{n-1}$. On the other hand, $\mathcal{P}_{n}^{<\infty} \subseteq{ }^{\perp_{1}}\left(\left(\mathcal{P}_{n}^{<\infty}\right)^{\perp_{1}}\right)=\mathcal{A}_{n}$. This implies that $M \in \mathcal{P}_{n-1}$, a contradiction.

In general, the tilting modules $T_{n}$ are infinitely generated. For artin algebras, we will characterize the case when $T_{n}$ can be taken finitely generated in Proposition 4.1 below.

The following example shows that the tilting modules $T_{n}$ need not be Extorthogonal.

Example 2.4. Let us consider the serial algebra $R$ of global dimension 2 given by the quiver $\bullet \stackrel{\alpha}{\longrightarrow} \stackrel{\beta}{\longrightarrow} \bullet \stackrel{\gamma}{\longrightarrow}$ - with the relation $\gamma \beta \alpha=0$. We number 
the vertices from left to right and denote by $P_{i}, S_{i}$ and $I_{i}$ the indecomposable projective, the simple, and the indecomposable injective corresponding to the $i$ th vertex, respectively. Then we see that we can choose $T_{1}=P_{1} \oplus P_{2} \oplus S_{2} \oplus$ $P_{2} / P_{4}$, while $T_{2}$ will be the minimal injective cogenerator of $\operatorname{Mod} R$. In particular, $\operatorname{Ext}_{R}^{1}\left(T_{2}, T_{1}\right) \neq 0$, because we have an almost split sequence $0 \longrightarrow P_{2} / P_{4} \longrightarrow$ $P_{1} \oplus S_{2} \longrightarrow I_{2} \longrightarrow 0$.

Observe that we get a large supply of tilting modules by varying the class $\mathcal{S}$ :

Remark 2.5. For all $0<i \leq n<\omega$, denote by $\left(\mathcal{A}_{n i}, \mathcal{B}_{n i}\right)$ the cotorsion pair cogenerated by the class $\Omega^{i-1}\left(\mathcal{P}_{n}^{<\infty}\right)$. By dimension shifting, we have $\mathcal{B}_{n i}=\left(\mathcal{P}_{n}^{<\infty}\right)^{\perp_{i}}$. Note that $\mathcal{B}_{n i} \subseteq \mathcal{B}_{n j}$ for $i \leq j, \mathcal{B}_{n i} \subseteq \mathcal{B}_{m i}$ for $m \leq n$, and $\mathcal{B}_{n i} \subseteq \mathcal{B}_{n+k, i+k}$ for all $k<\omega$.

Now, if $R$ is right coherent, then Lemma 2.2 for $\mathcal{S}=\Omega^{i-1}\left(\mathcal{P}_{n}^{<\infty}\right)$ yields a tilting module $T_{n i}$ of projective dimension at most $n-i+1$ such that $\mathcal{B}_{n i}=T_{n i}^{\perp}$. If $R$ is right noetherian and findim $R \geq n$, then we can show as in Proposition 2.3 that the projective dimension of $T_{n i}$ equals $n-i+1$.

In particular, it follows that the classes $\mathcal{B}_{n n}(n<\omega)$ form an increasing chain of tilting torsion classes in the sense of [6].

Our main result in this section characterizes the case of $\operatorname{fin} \operatorname{dim} R<\infty$ for right noetherian rings:

Theorem 2.6. Assume that $R$ is right noetherian. Then $\operatorname{findim} R<\infty$ if and only if there is a tilting module $T$ such that $\mathcal{B}=T^{\perp}$. In this case findim $R=\operatorname{pd} T$, and $T$ can be taken $\mathcal{P}^{<\infty}$-filtered.

Proof. The only-if-part is true for any right coherent ring by Proposition 2.3 for $n=$ findim $R$; that also gives $\operatorname{findim} R=\operatorname{pd} T$. The if-part holds by Lemma 2.2 for $\mathcal{S}=\mathcal{P}^{<\infty}$. Finally, Theorem 1.3 2) and Lemma 1.7(b) provide for a $\mathcal{P}^{<\infty}$-filtered module $D$ and a module $C \in \operatorname{Add} T$ such that $D=C \oplus T$. Then $D$ is also a tilting module with $\mathcal{B}=D^{\perp}$.

In particular cases, it is possible to compute the tilting module $T$ explicitly:

Example 2.7. (a) Let $R$ be a right noetherian ring of finite global dimension. Let $0 \longrightarrow R \longrightarrow I_{0} \longrightarrow I_{1} \longrightarrow \cdots \longrightarrow I_{n} \longrightarrow 0$ be the minimal injective resolution of $R$. Then $\bigoplus_{i<n} I_{i}$ is a tilting module as in Theorem 2.6.

Indeed, in this case, $\mathcal{P}<\infty=\bmod -R$; so $\mathcal{B}$ is the class of all injective modules. As mentioned in Section 1, the tilting module $T$ can be obtained by an iteration of special $\mathcal{B}$-preenvelopes of $R$, etc. In particular, the minimal injective resolution of $R$ yields a copy of $T$ as above.

(b) Let $R$ be a right coherent ring such that findim $R<\infty$ and $\mathcal{A} \cap \mathcal{B}$ consists of pure-injective modules. (Since $\mathcal{A} \cap \mathcal{B}=\operatorname{Add} T$, the latter condition is equivalent to assuming that $T$ is $\sum$-pure-injective.) Let $M$ be the direct sum of a representative set of all indecomposable modules from $\mathcal{A} \cap \mathcal{B}$. Then $M$ is a tilting module with $\mathcal{B}=M^{\perp}$.

This follows from Azumaya's Decomposition Theorem and from the fact that the $\sum$-pure-injective module $T$ is a direct sum of indecomposable summands with local endomorphism rings.

(c) Finally, we consider Cohen-Macaulay algebras and show that the dual of the dualizing module is a tilting module as in Theorem [2.6. 
Let $R$ be an artin algebra with the usual duality $D: \bmod R \rightarrow R \bmod$. Following [5], we call $R$ a Cohen-Macaulay algebra if there is a dualizing module $\omega$, that is, a faithfully balanced bimodule $R \omega_{R}$ which is a finitely generated strong cotilting module on both sides. Here a left module $M \in R \bmod$ is said to be a cotilting module if there is a tilting module $T \in \bmod R$ such that $M=D(T)$. Furthermore, $M$ is called a strong cotilting module if, in addition, the category $\mathcal{I}^{<\infty}$ of all finitely presented left $R$-modules of finite injective dimension coincides with the subcategory of $R \bmod$ consisting of all modules $X$ that have a finite add $M$-resolution $0 \longrightarrow$ $M_{n} \longrightarrow \cdots \longrightarrow M_{0} \longrightarrow X \longrightarrow 0$ with $M_{0}, \ldots, M_{n} \in \operatorname{add} M$.

Assume now that $R$ is a Cohen-Macaulay algebra with dualizing module $\omega$. We show that the finitely presented tilting module $T_{R}=D\left({ }_{R} \omega\right)$ satisfies $\mathcal{B}=T^{\perp}$. First of all, $\perp^{\perp}\left(\mathcal{I}^{<\infty}\right)={ }^{\perp} \omega$. In fact, the inclusion " $\subset$ " follows immediately from $\omega \in \mathcal{I}^{<\infty}$. Further, if ${ }_{R} Y \in{ }^{\perp} \omega$ and ${ }_{R} X \in \mathcal{I}^{<\infty}$, then we see by induction on the length of the add $\omega$-resolution of $X$ that $\operatorname{Ext}_{R}^{i}(Y, X)=0$ for any $i>0$.

Next, we show that the dual $D(X)$ of a module $X_{R} \in T^{\perp}$ is contained in ${ }^{\perp} \omega$. This follows from the well-known formula $\operatorname{Ext}_{R}^{i}(D(X), \omega) \cong D \operatorname{Tor}_{i}^{R}(D(X), D(\omega)) \cong$ $\operatorname{Ext}_{R}^{i}(D(\omega), X)$, where the second isomorphism holds because $D(\omega)$ is a finitely generated module and $R$ is noetherian.

Now we verify that every module $X_{R} \in T^{\perp}$ is contained in $\mathcal{B}$. Take a module $A_{R} \in \mathcal{P}^{<\infty}$. Of course, $D(A) \in \mathcal{I}^{<\infty}$; hence $\operatorname{Ext}_{R}^{i}(D(X), D(A))=0$ for any $i>0$ by the above computations, and the formula $\operatorname{Ext}_{R}^{i}(A, X) \cong D \operatorname{Tor}_{i}^{R}(D(X), A) \cong$ $\operatorname{Ext}_{R}^{i}(D(X), D(A))$ again gives the claim. Finally, since $T \in \mathcal{P}^{<\infty}$, we conclude that $\mathcal{B}=T^{\perp}$.

\section{A CRITERION FOR EQUALITy OF THE Finitistic Dimensions}

In this section, we give a criterion for validity of the First Finitistic Dimension Conjecture in terms of the tilting module $T$ considered in Theorem 2.6. The following general result will be crucial:

Proposition 3.1. Let $R$ be a ring and $M$ be a tilting module. Then the following two conditions are equivalent:

(1) the category Add $M$ is closed under cokernels of monomorphisms;

(2) $\mathcal{P}={ }^{\perp}\left(M^{\perp}\right)$.

These conditions imply Findim $R<\infty$.

If $M$ is finitely presented, then these conditions also imply

(3) $\mathcal{A}={ }^{\perp}\left(M^{\perp}\right)$.

If $M$ is finitely presented and $\sum$-pure-injective, then (3) implies (1); so all three conditions are equivalent.

Proof. Put $\mathcal{X}=M^{\perp}$.

Assume (1). By Theorem [1.8 we have ${ }^{\perp} \mathcal{X} \subseteq \mathcal{P}_{n}$, where $n$ is the projective dimension of $T$, and for any module $A \in \mathcal{P}$ there is an exact sequence $0 \longrightarrow A \longrightarrow$ $X \longrightarrow Y \longrightarrow 0$ with $X \in \mathcal{X}$ and $Y \in{ }^{\perp} \mathcal{X}$. Clearly, $X \in \mathcal{P}$. Let $m$ be the projective dimension of $X$. By Lemma 1.7(c), there is a long exact sequence

$$
0 \longrightarrow M_{m+1} \longrightarrow M_{m} \longrightarrow M_{m-1} \longrightarrow \cdots \longrightarrow M_{0} \longrightarrow X \longrightarrow 0
$$

with $M_{i} \in \operatorname{Add} M$ for all $i=0, \ldots, m+1$. Using the assumption, and induction, we obtain $X \in \operatorname{Add} M \subseteq{ }^{\perp} \mathcal{X}$. Since ${ }^{\perp} \mathcal{X}$ is resolving, we infer $A \in{ }^{\perp} \mathcal{X}$. This proves that $\mathcal{P} \subseteq{ }^{\perp} \mathcal{X}$, and hence $\mathcal{P}={ }^{\perp} \mathcal{X}$. 
Assume (2). Then $\operatorname{Add} M=\mathcal{P} \cap \mathcal{X}$ by Lemma 1.7(b). So Add $M$ is closed under cokernels of monomorphisms since $\mathcal{P}$ and $\mathcal{X}$ share this property. Moreover, $\mathcal{P}$ is closed under arbitrary direct sums; hence there is $n<\omega$ such that $\mathcal{P}=\mathcal{P}_{n}$, and Findim $R<\infty$.

Now, assume $M$ is finitely presented. Then $M \in \mathcal{A}$, and hence ${ }^{\perp} \mathcal{X} \subseteq \mathcal{A}$. If (2) holds, we also have $\mathcal{A} \subseteq \mathcal{P}_{n}=\mathcal{P}={ }^{\perp} \mathcal{X}$, which proves (3).

Finally, let $M$ be finitely presented and $\sum$-pure-injective. Assume (3).

We first show that every monomorphism in add $M$ splits. Indeed, let $0 \longrightarrow A \stackrel{f}{\longrightarrow}$ $B \longrightarrow C \longrightarrow 0$ be a short exact sequence with $A$ and $B$ in add $M$. By assumption, $\operatorname{add} M \subset \mathcal{B} \cap \mathcal{P}^{<\infty}$. Since $\mathcal{P}<\infty$ is closed under cokernels of monomorphisms, we have $C \in \mathcal{P}^{<\infty} \subset \mathcal{A}$ and $A \in \mathcal{B}$; so $\operatorname{Ext}_{R}^{1}(C, A)=0$ and $f$ splits.

Since $M$ is $\Sigma$-pure-injective, we infer that any monomorphism in Add $M$ is pure, and hence splits; cf. [13, Observation 3.3]. So (1) holds true.

Now, we easily obtain a characterization for $\mathcal{A}=\mathcal{P}$ in the right coherent case:

Theorem 3.2. Let $R$ be a right coherent ring. The following are equivalent:

(1) findim $R<\infty$, and every module of finite projective dimension is a direct summand of a $\mathcal{P}<\infty$-filtered module.

(2) There is a tilting module $T$ such that $\mathcal{B}=T^{\perp}$ and $\operatorname{Add} T$ is closed under cokernels of monomorphisms.

(3) $\mathcal{A}=\mathcal{P}$.

Under any of these conditions we have Findim $R=\operatorname{findim} R<\infty$.

Proof. (1) is equivalent to (3): The second condition of (1) is equivalent to $\mathcal{P} \subseteq$ $\mathcal{A}$ by Theorem 1.3 2). Further, findim $R<\infty$ implies $\mathcal{A} \subseteq \mathcal{P}$ by Lemma 1.4 and conversely, $\mathcal{A}=\mathcal{P}$ implies that $\mathcal{P}$ is closed under direct sums and therefore Findim $R<\infty$.

The equivalence of (1) and (2) now follows from Propositions 2.3 and 3.1 Finally, condition (1) obviously implies that $\operatorname{Fin} \operatorname{dim} R=$ findim $R$.

Corollary 3.3. Let $R$ be a right coherent and right perfect ring. Then the following statements are equivalent:

(1) $\mathcal{A}=\mathcal{P}$;

(2) $\mathcal{A}$ is closed under direct limits, and a module has finite projective dimension if and only if it is a direct limit of finitely presented modules having finite projective dimension.

Proof. Recall that every module in $\mathcal{A}$ is a direct limit of modules from $\mathcal{P}<\infty$ by Lemma 2.1. If $\mathcal{A}=\mathcal{P}$, then $\mathcal{P}=\mathcal{P}_{n}$ for some $n \geq 0$, and since $\mathcal{P}_{n}$ is closed under direct limits over any right perfect ring, we conclude that $\mathcal{A}$ coincides with the class of all direct limits of modules from $\mathcal{P}^{<\infty}$. The converse implication follows immediately from the fact that $\mathcal{P}<\infty$ is contained in $\mathcal{A}$.

Next, we discuss when $\mathcal{A}$ is closed under direct limits. Recall that a module $M$ with Add $M$ being closed under products is said to be product-complete; see [14].

Theorem 3.4. Let $R$ be a two-sided coherent and right perfect ring, and assume that the modules of finite projective dimension coincide with the direct limits of finitely presented modules having finite projective dimension. Then the following statements are equivalent:

(1) $\mathcal{A}$ is closed under direct limits; 
(2) Findim $R<\infty$, and $\mathcal{B}=T^{\perp}$ for a product-complete tilting module $T$;

(3) Findim $R<\infty$, and $\mathcal{B}=T^{\perp}$ for a $\sum$-pure-injective tilting module $T$.

Proof. (1) implies (2): We know from Corollary 3.3 and Theorem 3.2 that $\mathcal{A}=\mathcal{P}$ and Findim $R<\infty$. By assumption, the class $\mathcal{P}=\mathcal{P}_{n}$ is closed under products; so $\operatorname{Add} T=\mathcal{P} \cap \mathcal{B}$ has the same property, and $T$ is product-complete.

(2) implies (3) since any product-complete module is $\sum$-pure-injective.

(3) implies (1): Since Findim $R<\infty$, we know from [8, Theorem 7.4.6] that $\left(\mathcal{P}, \mathcal{P}^{\perp}\right)$ is a cotorsion pair. We now show that it coincides with our cotorsion pair $(\mathcal{A}, \mathcal{B})$. Then Corollary 3.3 will yield $(1)$.

As mentioned in Section 1, the tilting module $T$ with $\mathcal{B}=T^{\perp}$ can take the form $T=\bigoplus_{i \leq m} B_{i}$, where $0 \longrightarrow R \stackrel{f_{0}}{\longrightarrow} B_{0} \stackrel{f_{1}}{\longrightarrow} B_{1} \longrightarrow \cdots \stackrel{f_{m}}{\longrightarrow} B_{m} \longrightarrow 0$ is a long exact sequence obtained by an iteration of special $\mathcal{B}$-preenvelopes.

By Lemma 1.7 (b), also this special choice of $T$ must then be $\sum$-pure-injective. Thus Add $T$ consists of pure-injective modules and is contained in $\mathcal{B}=\left(\mathcal{P}^{<\infty}\right)^{\perp}$. From the assumption and Lemma 1.6. we then infer that Add $T$ is even contained in $\mathcal{P}^{\perp}$

In particular, we deduce that $B_{i} \in \mathcal{P}^{\perp}$ for all $i \leq m$. Moreover, the cokernels Coker $f_{i}$ are in $\mathcal{A} \subseteq \mathcal{P}$. So, the above long exact sequence can also be viewed as an iteration of special $\mathcal{P}^{\perp}$-preenvelopes. Now, taking $\mathcal{X}=\mathcal{P}^{\perp}$ in the proof of Theorem 1.8 (cf. [1, Theorem 4.1]), we get $T^{\perp}=\mathcal{P}^{\perp}$, and $\mathcal{A}=\mathcal{P}$.

The results above lead to the question of when are all modules from $\mathcal{P}_{n}$ direct limits of modules from $\mathcal{P}_{n}^{<\infty}$. A sufficient condition in the semi-perfect case is as follows:

Lemma 3.5. Let $R$ be a ring such that each projective module is a direct sum of finitely generated modules (e.g., let $R$ be semi-perfect). Let $0<n<\omega$. Assume that each module from $\mathcal{P}_{n-1}$ is a directed union of modules from $\mathcal{P}_{n-1}^{<\infty}$. Then each module from $\mathcal{P}_{n}$ is a direct limit of modules from $\mathcal{P}_{n}^{<\infty}$.

Proof. Let $M \in \mathcal{P}_{n}$. So there is an exact sequence $0 \rightarrow N \hookrightarrow \bigoplus_{\beta<\lambda} P_{\beta} \rightarrow M \rightarrow 0$ where $N \in \mathcal{P}_{n-1}$ and all $P_{\beta}(\beta<\lambda)$ are finitely generated and projective. By assumption, $N=\bigcup_{\alpha \in I} N_{\alpha}$ for a directed set $(I, \leq)$ and for $N_{\alpha} \in \mathcal{P}_{n-1}^{<\infty}(\alpha \in I)$. Without loss of generality, we assume that $\omega \leq \operatorname{card}(I)$.

For each $\alpha \in I$, let $L_{\alpha}$ be the smallest finite subset of $\lambda$ such that $N_{\alpha} \subseteq$ $\bigoplus_{\beta \in L_{\alpha}} P_{\beta}$. Then the short exact sequences $0 \rightarrow N_{\alpha} \hookrightarrow \bigoplus_{\beta \in L_{\alpha}} P_{\beta} \rightarrow M_{\alpha} \rightarrow 0$ $(\alpha \in I)$ form a direct system: for $\alpha \leq \alpha^{\prime} \in I$, the direct system map $f_{\alpha \alpha^{\prime}}$ is defined as the triple $(\mu, \nu, \pi)$ where $\mu: N_{\alpha} \hookrightarrow N_{\alpha^{\prime}}$ and $\nu: \bigoplus_{\beta \in L_{\alpha}} P_{\beta} \hookrightarrow \bigoplus_{\beta \in L_{\alpha^{\prime}}} P_{\beta}$ are the inclusions, and $\pi: M_{\alpha} \rightarrow M_{\alpha^{\prime}}$ is the induced map. Observe that $M_{\alpha} \in \mathcal{P}_{n}^{<\infty}$ for all $\alpha \in I$.

Put $L=\bigcup_{\alpha \in I} L_{\alpha}$. Then the direct limit of the direct system defined above is the exact sequence $0 \rightarrow N \hookrightarrow \bigoplus_{\beta \in L} P_{\beta} \rightarrow \lim _{\alpha} M_{\alpha} \rightarrow 0$. So $M \cong \lim _{\alpha} M_{\alpha} \oplus$ $\bigoplus_{\beta<\lambda, \beta \notin L} P_{\beta}$ is a direct limit of elements from $\mathcal{P}_{n}^{<\infty}$.

As a first corollary, we obtain a result proved by Huisgen-Zimmermann in the finite-dimensional algebra case (cf. [12, Observation 5])

Corollary 3.6. Let $R$ be a semi-perfect ring and $M \in \mathcal{P}_{1}$. Then $M$ is a direct limit of a direct system of modules from $\mathcal{P}_{1}^{<\infty}$. 
Proof. Since $R$ is semi-perfect, each module from $\mathcal{P}_{0}$ is a direct sum of cyclic modules, hence a directed union of a direct system of modules from $\mathcal{P}_{0}^{<\infty}$, and Lemma 3.5 applies.

Corollary 3.7. Let $R$ be a two-sided coherent and right perfect ring such that Findim $R=1$. Then $\mathcal{A}=\mathcal{P}$ iff all modules in $\mathcal{A} \cap \mathcal{B}$ are pure-injective.

Proof. This follows immediately from Theorem 3.4 and Corollaries 3.3 and 3.6

Unfortunately, even for finite-dimensional algebras, $M \in \mathcal{P}_{1}$ need not be a directed union of elements of $\mathcal{P}_{1}^{<\infty}$, and the conclusion of Lemma 3.5 may fail for $n \geq 2$. The relevant example is due to Smalø [18]:

Example 3.8. For each $m \geq 2$, there exists a finite-dimensional algebra $R$ over an algebraically closed field $k$ and a module $M \in \mathcal{P}_{m}$ such that $M$ is not a direct limit of a direct system of modules from $\mathcal{P}_{m}^{<\infty}$. Indeed, consider the finite-dimensional algebra $R$ constructed in [18, $\S 1$ ] for $n=2$. So findim $R=1$ and $\operatorname{Findim} R=2$ by [18, Theorem 1.1]. Then a module $M$ of projective dimension 2 cannot be a direct limit of a direct system of modules from $\mathcal{P}_{m}^{<\infty}\left(=\mathcal{P}_{1}^{<\infty}\right)$ since $\mathcal{P}_{1}$ is closed under direct limits. Let $N$ be the first syzygy module of $M$; so $N \in \mathcal{P}_{1}$. By Lemma 3.5. $N$ is not a directed union of elements of $\mathcal{P}_{1}^{<\infty}$.

\section{Finitistic Dimensions of ARTin ALGEBRAS}

In this section, $R$ will denote an artin algebra. We will prove that the tilting module $T$ in Theorem 2.6 can be chosen finitely presented if and only if the category $\mathcal{P}<\infty$ is contravariantly finite.

Recall that a subcategory $\mathcal{M}$ of $\bmod R$ is contravariantly (respectively, covariantly) finite in $\bmod R$ if every finitely presented module has an $\mathcal{M}$-precover (respectively, an $\mathcal{M}$-preenvelope). In this case, it is well known that the $\mathcal{M}$-precovers $(\mathcal{M}$-preenvelopes) have minimal versions which are special.

Proposition 4.1. Let $R$ be an artin algebra. Let $\mathcal{S}$ be a syzygy-closed subclass of $\mathcal{P}^{<\infty}$. Denote by $(\mathcal{U}, \mathcal{V})$ the cotorsion pair cogenerated by $\mathcal{S}$. The following are equivalent:

(1) $\mathcal{U} \cap \bmod R$ is contravariantly finite in $\bmod R$;

(2) there exists a finitely presented tilting module $T$ such that $\mathcal{V}=T^{\perp}$.

Proof. That (2) implies (1) is due to Auslander and Reiten. In fact, we know from [5, $\S 5]$ that $\mathcal{V} \cap \bmod R$ is coresolving and covariantly finite in $\bmod R$. Then $\mathcal{U} \cap \bmod R$ is contravariantly finite by Remark 1.2

Assume (1). From $\mathcal{S} \subseteq \mathcal{P}^{<\infty}$ we deduce $\mathcal{U} \subseteq \mathcal{A}$; so $\mathcal{U} \cap \bmod R \subseteq \mathcal{A} \cap \bmod R=$ $\mathcal{P}^{<\infty}$ by [20, Corollary 2.6]. Following [5, 3.9], there even exists $n<\omega$ such that $\mathcal{U} \cap \bmod R \subseteq \mathcal{P}_{n}^{<\infty}$. Since $\mathcal{S} \subseteq \mathcal{U} \cap \bmod R$, we see that $\mathcal{V}=M^{\perp}$ for a module $M$ of projective dimension at most $n$. Remark 1.2 gives that every finitely presented module $X$ has a special $\mathcal{V}$-preenvelope $0 \longrightarrow X \longrightarrow V \longrightarrow U \longrightarrow 0$ where $V \in$ $\mathcal{V} \cap \bmod R$ and $U \in \mathcal{U} \cap \bmod R$. We can then construct a long exact sequence $0 \rightarrow R \rightarrow V_{0} \rightarrow V_{1} \rightarrow \cdots \rightarrow V_{n-1} \rightarrow V_{n} \rightarrow 0$ with $V_{0}, \ldots, V_{n-1} \in \mathcal{V} \cap \bmod R$ and all cokernels in $\mathcal{U} \cap \bmod R$. By dimension shifting, we see that $V_{n}$ is contained in $M^{\perp}$, hence in $\mathcal{V} \cap \bmod R$. The same argument as in the proof of [1, 4.1] now shows that $T=\bigoplus_{i=0}^{n} V_{i}$ is a finitely presented tilting module such that $\mathcal{V}=T^{\perp}$. 
Theorem 4.2. Let $R$ be an artin algebra. The following conditions are equivalent:

(1) $\mathcal{P}<\infty$ is contravariantly finite in $\bmod R$;

(2) there is a finitely presented tilting module $T$ such that $\mathcal{B}=T^{\perp}$.

Proof. By 20, Corollary 2.6], $\mathcal{P}^{<\infty}=\mathcal{A} \cap \bmod R$ over any right noetherian ring. So the equivalence follows from Proposition 4.1 for $\mathcal{S}=\mathcal{P}^{<\infty}$.

If $R$ is an artin algebra such that $\mathcal{P}<\infty$ is contravariantly finite in $\bmod R$, then it was shown by Huisgen-Zimmermann and Smalø in 13] that the First and the Second Finitistic Dimension Conjectures hold true. The hard core of their proof consisted of showing directly that $\mathcal{P}$ coincides with the class of all direct limits of modules in $\mathcal{P}^{<\infty}$. The following is a refinement of that result:

Corollary 4.3. If $R$ is an artin algebra such that $\mathcal{P}<\infty$ is contravariantly finite in $\bmod R$, then every module of finite projective dimension is a direct summand of a $\mathcal{P}^{<\infty}$-filtered module. In particular, Findim $R=\operatorname{findim} R<\infty$.

Proof. By Theorem 4.2, there is a finitely presented tilting module $T$ such that $\mathcal{B}=T^{\perp}$. Since $T$ is $\sum$-pure-injective, Proposition 3.1 and Theorem 3.2 yield $\mathcal{A}=\mathcal{P}$ and Findim $R=$ findim $R<\infty$.

Corollary 4.4. Let $R$ be an artin algebra. The following conditions are equivalent:

(1) $\mathcal{P}^{<\infty}$ is contravariantly finite in $\bmod R$;

(2) there is a finitely presented tilting module $T$ such that $\mathcal{P}^{\perp}=T^{\perp}$;

(3) there is a finitely presented tilting module $T$ such that $T^{\perp} \subset M^{\perp}$ for all tilting modules $M$.

Proof. (1) implies (2) is just Theorem 4.2 together with the condition $\mathcal{A}=\mathcal{P}$ shown in Corollary 4.3 .

(2) implies (3) is clear since every tilting module $M$ belongs to $\mathcal{P}$, and hence $\mathcal{P}^{\perp} \subseteq M^{\perp}$.

(3) implies (1): In particular, it follows that $T^{\perp}$ is contained in all $T_{n}{ }^{\perp}=\mathcal{B}_{n}$ considered in Proposition 2.3 Hence $T^{\perp}$ is contained in their intersection $\mathcal{B}$. Since $T \in \mathcal{P}^{<\infty}$, we conclude that $\mathcal{B}=T^{\perp}$, and (1) is verified by Theorem 4.2.

Remark 4.5. Applying Proposition 4.1 to $\mathcal{S}=\Omega^{i-1}\left(\mathcal{P}_{n}^{<\infty}\right)$ with $0<i \leq n<\omega$, we get the corresponding statements for the cotorsion pairs $\left(\mathcal{A}_{n i}, \mathcal{B}_{n i}\right)$ considered in Remark 2.5] More precisely, the category $\mathcal{A}_{n i} \cap \bmod R$ is contravariantly finite in $\bmod R$ if and only if there is a finitely presented tilting module $T_{n i}$ such that $\mathcal{B}_{n i}=T_{n i}^{\perp}$. (By [20, Corollary 2.4], this is also equivalent to the following assertion: "For each simple module $S$ there is a special $\mathcal{A}_{n i}$-precover of $S, f: A \rightarrow S$, such that $A \in \bmod R$.") For $i=1$, we infer that the class $\mathcal{P}_{n}^{<\infty}$ is contravariantly finite in $\bmod R$ iff there is a finitely presented tilting module $T_{n}$ such that $\mathcal{B}_{n}=T_{n}^{\perp}(n<\omega)$.

\section{REFERENCES}

[1] L. Angeleri Hügel, F. U. Coelho, Infinitely generated tilting modules of finite projective dimension, Forum Math. 13 (2001), 239-250. MR 2002b:16009

[2] L. Angeleri Hügel, F. U. Coelho, Infinitely generated complements to partial tilting modules, Math. Proc. Cambridge Philos. Soc. 132 (2002), 88-95.

[3] L. Angeleri Hügel, A. Tonolo, J. Trlifaj, Tilting preenvelopes and cotilting precovers, Algebras and Representation Theory 4 (2001), 155-170. MR 2002e: 16010

[4] M. Auslander, Functors and morphisms determined by objects, in Representation Theory of Algebras, Lecture Notes in Pure Appl. Math.37, M.Dekker, New York, 1978, pp. 1-244. MR 58:844 
[5] M. Auslander, I. Reiten, Applications of contravariantly finite subcategories, Adv. Math. 86 (1991), 111-152. MR 92e:16009

[6] R. Colpi, J. Trlifaj, Tilting modules and tilting torsion classes, J. Algebra 178 (1995), 614634. MR 97e: 16003

[7] W. Crawley-Boevey, Infinite-dimensional modules in the representation theory of finitedimensional algebras, Canad. Math. Soc. Conf. Proc.23(1998), 29-55. MR 99m:16016

[8] E. E. Enochs, O. M. G. Jenda, Relative Homological Algebra, de Gruyter, Berlin, 2000. MR 2001h:16013

[9] P. Eklof, J. Trlifaj, How to make Ext vanish, Bull. London Math. Soc. 33 (2001), 41-51. MR 2001i:16015

[10] R. Göbel, S. Shelah, Cotorsion theories and splitters, Trans. Amer. Math. Soc. 352 (2000), 5357-5379. MR 2001b:20098

[11] D. Happel, C. M. Ringel, Tilted algebras, Trans. Amer Math. Soc. 274 (1982), 399-443. MR 84d:16027

[12] B. Huisgen-Zimmermann, Homological assets of positively graded representations of finite dimensional algebras, Canad. Math. Soc. Conf. Proc.14(1993), 463-475.

[13] B. Huisgen-Zimmermann, S. O. Smalø, A homological bridge between finite and infinite dimensional representations, Algebras and Representation Theory 1 (1998), 169-188. MR 2000b:16014

[14] H. Krause, M. Saorín, On minimal approximations of modules, In: Trends in the representation theory of finite dimensional algebras (ed. by E. L. Green and B. Huisgen-Zimmermann), Contemp. Math. 229 (1998) 227-236. MR 99m:16002

[15] H. Lenzing, Homological transfer from finitely presented to infinite modules, Lecture Notes in Math. 1006 (1983), 734-761. MR 85f:16034

[16] Y. Miyashita, Tilting modules of finite projective dimension, Math. Z. 193 (1986), 113-146. MR 87m:16055

[17] L. Salce, Cotorsion theories for abelian groups, Symposia Math. XXIII (1979), 11-32. MR 81j:20078

[18] S. O. Smalø, Homological differences between finite and infinite dimensional representations of algebras, Trends in Math., Birkhäuser, Basel, 2000, 425-439. MR 2001i:16013

[19] J. Trlifaj, Cotorsion theories induced by tilting and cotilting modules, Abelian Groups, Rings and Modules, Contemp. Math. 273 (2001), 285-300. MR 2001m:16012

[20] J. Trlifaj, Approximations and the little finitistic dimension of artinian rings, J. Algebra 246 (2001), 343-355.

Mathematisches Institut der Universität, Theresienstrasse 39, D-80333 München, GERManY

E-mail address: angeleri@rz.mathematik.uni-muenchen.de

Current address: Universitat Autònoma de Barcelona, Departament de Matemàtiques, E08193 Bellaterra (Barcelona), Spain

Katedra algebry MFF UK, Sokolovská 83, 18675 Prague 8, Czech Republic

E-mail address: trlifaj@karlin.mff.cuni.cz 